\title{
New Researches of State Key Laboratories in Analytical Chemistry
}

\author{
Huangxian $\mathrm{Ju}^{1 *}$, Weihong $\mathrm{Tan}^{2}$, Lehui $\mathrm{Lu}^{3}$, Maili $\mathrm{Liu}^{4}$ \\ ${ }^{1}$ Department of Chemistry, Nanjing University, Nanjing 210093, China \\ ${ }^{2}$ College of Chemistry and Chemical Engineering, Hunan University, Changsha 410082, China \\ ${ }^{3}$ Changchun Institute of Applied Chemistry, Chinese Academy of Sciences, Changchun 130022, China \\ ${ }^{4}$ Wuhan Institute of Physics and Mathematics, Chinese Academy of Sciences, Wuhan 430071, China
}

In the past two decades, as the eyes of fundamental scientific research and social activities relative to human security and health, analytical chemistry has made great progress in both analytical methodologies and detection instruments by integrating multidisciplinary research achievements. Four State Key Laboratories were successively established in this rapidly developing discipline. In order to publish some of the excellent results together, we have organized this special topic with 10 Articles and Reviews from these State Key Laboratories.

State Key Laboratory of Analytical Chemistry for Life Science (Nanjing University) was promoted from the National Key Discipline in Analytical Chemistry and former Key Laboratory of Analytical Chemistry for Life Science (Ministry of Education of China, MOE), and approved by the Ministry of Science and Technology of China (MOST) on October 13, 2011.

This discipline was authorized to offer doctoral degree program in 1981, and approved as National Key Disciplines in 1987. Since the beginning of the 1990's, its research activities have focused on solving the key problems of analytical chemistry in life sciences. "Key Laboratory of Analytical Chemistry for Life Science" was approved on March 5, 2004 by MOE. A strong research team with vigor and creativity was recognized as "Creative Research Group" by the National Natural Science Foundation of China in 2005. It was recommended by MOE as a candidate for a State Key Laboratory in November 2010, which was approved in 2011 and then certified in December 2013 by MOST.

This laboratory focuses mainly on the fundamental research of the methodologies of analytical chemistry for life substances, aiming at scientific frontiers and priorities of national interests. The main research areas include surface and interfacial behaviors of biomolecules, biomolecular and

*Corresponding author (email: hxju@ nju.edu.cn) biomimic recognition, bioanalysis in micro/nano scale, identification and detection of biomarkers as well as apparatus development.

State Key Laboratory of Chemo/Biosensing and Chemometrics (Hunan University) was approved by MOST in 2001. Its primary research areas include but not limited to bioanalytical chemistry, Chemo/Biosensing, chemometrics, chemical biology, nanomedicine as well as analytical instrumentation. The laboratory has around 65 permanent members, including 3 members of the Chinese Academy of Sciences, 9 awardees of National Natural Science Foundation of China (NSFC) for Distinguished Young Scholars. Recently, this laboratory has recruited a supertalent team headed by Professor Chad Mirkin from Northwestern University in the US to launch an "Institute of Chemical Biology and Nanomedicine". The group is recruiting young faculty members from all over the world.

This laboratory has made great advancements in scientific research and academic exchanges in the past five years. Over 300 projects from the national and the provincial governments have been accomplished. Meanwhile, more than 1300 SCI papers have been published. The members of the laboratory have won two second prizes of National Natural Science Award in the last five years and numerous other Science and Technology awards. The laboratory holds the series of International Symposium on Bioanalysis, Biomedical Engineering and Nanotechnology every other year.

State Key Laboratory of Electroanalytical Chemistry (Changchun Institute of Applied Chemistry, Chinese Academy of Sciences) was promoted from the former Open Laboratory of Electroanalytical Chemistry of the Chinese Academy of Sciences and approved by MOST in July, 2001.

This laboratory focuses mainly on the frontiers of analytical chemistry and electroanalytical chemistry. Aiming at the original innovation, it strives for significant theoretical breakthrough, develops new analytical principle and estab- 
lishes in situ, in vivo, real-time, online method with high sensitivity and selectivity and multi-parameters. At the same time, it is more important to design new analytical instruments and cultivate outstanding talents in the process of research. The strategic goal of the laboratory is to construct an advanced base on fundamental research and talent training and to grow up the pioneer of electroanalytical chemistry in theory, method and technology.

In the past five years, the laboratory has won secondclass prize of National Natural Science Award twice, first-class prize of Jilin Province Natural Science Award twice. The laboratory boasts two academicians of Chinese Academy of Sciences, two academicians of the Academy of Sciences for the Developing World.

State Key Laboratory of Magnetic Resonance and Atomic and Molecular Physics (Wuhan Institute of Physics and Mathematics, Chinese Academy of Sciences) is one of the first batch of 18 laboratories of the CAS, open to the public. In 1986, it was approved by the National Planning Committee to build as a State Key Laboratory. In December 1988, the construction was passed. In 1999, it was included in the "Innovation Engineering Plan" of the CAS. Through more than ten-year construction and development, the foundation of the laboratory is now completed as a Chinese research basis of magnetic resonance and atomic and molecular physics of worldwide level. Part of its projects stepped into the world top frontier.

The main research fields in this laboratory are magnetic resonance and atomic and molecular physics. The research interest is to study the atomic and molecular structure, dynamics, and their environmental effects by the resonance interactions of electromagnetic waves (from radio to optical frequencies) with the atomic and molecular systems, including magnetic resonance imaging, the localized spectroscopy of magnetic resonance and its application in biology and life science; NMR methods in the study of structure dynamics and interaction of biological molecular; spin relaxation, molecular diffusion, chemical exchange and structure; solid state high resolution NMR and its application; cold atomic physics, the theory and experiment of quantum communication; trapping of atoms and ions and atomic hyperfine structure; atomic characteristics in strong external fields; laser spectroscopy and its applications; and high quality atomic frequency standard.

We hope this special topic could provide valuable references to the related researchers and play an active role in promoting the research work on Analytical Chemistry. Finally, we thank all the authors for their scientific contributions to this special topic and the referees for their careful reviewing of the submitted manuscripts. We also thank the editor Li Fu for her help in dealing with the manuscripts and organizing this special topic.

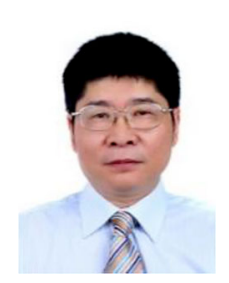

Prof. Huangxian Ju received his B.S., M.S and Ph.D degrees (1982-1992), and became an associate professor (1993) and professor (1999) in Nanjing University. He won the National Funds for National Distinguished Young Scholars in 2003, and was selected as a Changjiang Professor in 2007, a "973" chief scientist in 2009, Fellows of the ISE and the Royal Society of Chemistry in 2015. He is the director of State Key Laboratory of Analytical Chemistry for Life Science. His research interest focuses on signal amplification and life analytical chemistry. He has published 552 papers with an h-index of 75 (Google h-index 82).

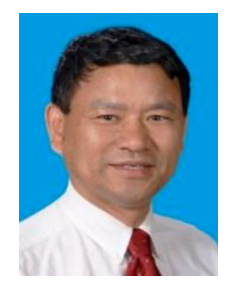

Prof. Weihong Tan received his Ph.D. in physical chemistry at the University of Michigan in the US. He has been a professor at the University of Florida since 1996. Since 2010, he has been the Director of the State Key Laboratory of Chemo/Biosensing and Chemometrics at Hunan University. He was elected to the Chinese Academy of Sciences in 2015 and a Fellow of the American Association for the Advancement of Science in 2005. Professor Tan's research is in bioanalytical chemistry, chemical biology and nanomedicine.

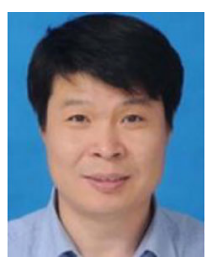

Prof. Lehui Lu received his M.S. (2000) and Ph.D. (2003) from the Changchun Institute of Applied Chemistry, Chinese Academy of Sciences (CIACCAS). He carried out his postdoctoral research at Hamburg University (2003-2005) and Kwansei Gakuin University (2005-2007). He is currently a professor at CIACCAS, where he is also the director of the State Key Laboratory of Electroanalytical Chemistry. His research focuses on nano-structured functional materials for energy, biomedical and environmental related applications.

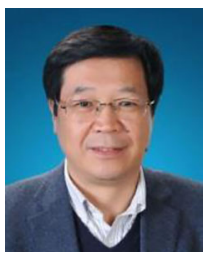

Prof. Maili Liu is director of the State Key Laboratory of Magnetic Resonance and Atomic and Molecular Physics, graduated from Northwest University in 1982, and received his Ph.D. from University of London (UK) in 1996. He won the National Funds for National Distinguished Young Scholars in 1999, and was a "973" chief scientist. His research interest is in development of novel NMR approaches for biological studies, including solvent suppression, complex and overlapped NMR spectra simplification, and study of bio-molecular structures, interaction and dynamics. 